\title{
Investigation of nanoporous material under quasi-equilibrium conditions
}

\author{
O.V. Yushchenko, T.I. Zhylenko \\ Sumy State University, 2 Rimskii-Korsakov St., 40007 Sumy, Ukraine
}

Received October 16, 2012, in final form December 5, 2012

Based on the three parametric Lorenz system, a model was developed that permits to describe the behavior of the plasma-condensate system near phase equilibrium in a self-consistent way. Considering the effect of fluctuations of the growth surface temperature, the evolution equation and the corresponding Fokker-Planck equation were obtained. The phase diagram is built which determines the system parameters corresponding to the regime of the porous structure formation.

Key words: self-organization, supersaturation, phase equilibrium, condensation, phase diagram

PACS: $64.70 . f m, 68.43 . \mathrm{Hn}, 05.10 . \mathrm{Gg}$

\section{Introduction}

Nowadays, modern nanotechnologies are developed by using a variety of methods, one of which is the condensation process in the steady state close to phase equilibrium. This method makes it possible to obtain various structures of a condensate, fractal surfaces, porous structures, etc. [1, 2]. The principal feature of this condensation process is the plasma-condensate system being close to phase equilibrium. Consequently, the adsorbed atoms are arranged on the active centers of crystallization forming structures with different architectures. In quasi-equilibrium conditions for continuous copper condensation of about 7 hours, the formation of highly porous structures, whiskers, and some intermediate structures (fibrous structures with alternating crystalline and porous parts) was observed. Of particular interest is the structure shown in figure 1 which unlike ordinary single crystals, is realized under unstable temperature regime.

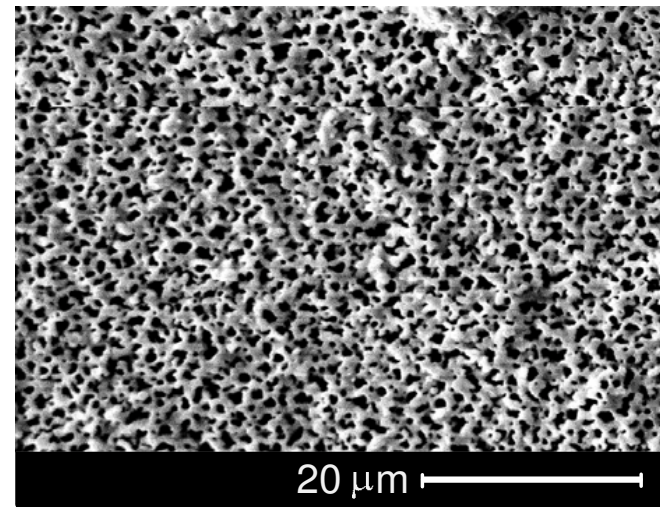

Figure 1. Porous structure of the copper, obtained by spray-deposited material at high discharge power [1, 2].
These structures may be of great practical interest. For example, they can be used as a molecular sieve.

However, a question arises regarding the reasons of holding the plasma-condensate system near phase equilibrium. Taking into account a universal nature of the condensation process, we assumed earlier [2-5] that it is caused by self-organization of a multi-phase plasma-condensate system. From the physical point of view, the mentioned selforganization is explained by an increase of the energy of the adsorbed atoms which results in the temperature increase of the growth surface under the effect of the plasma within the condensation process. On the other hand, an increase of the growth surface temperature is compensated by the desorption flow of the adsorbed atoms, which are 
responsible for supersaturation. As a result, within the framework of synergetic ideology [6], our consideration is based on the three-parameter Lorenz system [7, 8].

The paper is organized as follows. In section 2 the self-organized system that forms the basis of our consideration is described. Section 3 discusses the statistical analysis of the motion equation. The stationary solution of the Fokker-Planck equation is considered in section 4 General conclusions are presented in section 5 .

\section{Basic equations}

Considering that a quasi-equilibrium condensation is provided on the growth surface due to a selfconsistent development of the processes in the plasma volume, we will further use a three-dimensional (volume) concentration of the condensate $N$ and a two-dimensional (surface) concentration $n \equiv N a$. Here $a$ is a scale factor, which plays the role of the lattice parameter and its value will be determined below.

For a given value of the equilibrium concentration $n_{\mathrm{e}}$, the increasing supersaturation $n-n_{\mathrm{e}}$ is provided by the diffusion component defined by the Onsager relation [9, 10] for the adsorption flow

$$
J_{\text {ad }} \equiv D|\nabla N| \simeq \frac{D}{\lambda}\left(N_{\text {ac }}-N\right) \text {. }
$$

It takes into account that the main decrease in the concentration value takes place near the cathode layer, whose thickness is determined by the screening length $\lambda$. The latter and the diffusion coefficient $D$ are given by the equations [9, 10]

$$
\lambda^{2}=\frac{\varepsilon T_{\mathrm{p}}}{4 \pi e^{2} N_{\mathrm{i}}}, \quad D=\frac{\sigma T_{\mathrm{p}}}{e^{2} N_{\mathrm{i}}},
$$

where $\varepsilon, \sigma$ are the dielectric permittivity and the conductivity of the plasma, respectively, $T_{\mathrm{p}}$ is its temperature measured in the energy units; $e, N_{\mathrm{i}}$ are the charge and the total concentration of the ions of the deposited substance and the inert gas.

In the second relation (2.1), it is considered that at the upper boundary layer of the cathode the volume concentration of the deposited atoms is reduced to the accumulated value $N_{\mathrm{ac}}$, and the lower boundary of this layer presents the growth surface, near which the concentration of atoms is $N$.

A decrease of supersaturation $n-n_{\mathrm{e}}$ is ensured by the desorption flow $\mathbf{J}$, which is directed up from the growth surface, so that $J<0$, while the value of the adsorption flow $J_{\text {ad }}>0$. In case there is no condensate (when all the adsorbed atoms have evaporated from the substrate), the condition $J=-J_{\mathrm{ad}}$ is performed for the desorption component. Here, the accumulated flow $J_{\text {ad }}$ is defined by the equation 2.1), where $N=N_{\mathrm{e}}$. The diffusion changes of the concentration $N$ of the deposited atoms are presented by the continuity equation $\dot{n} / a+\nabla \mathrm{J}_{\mathrm{ad}}=0$. Here, the point over $n$ denotes the differentiation with respect to time and the source effect is given by the estimate

$$
\left|\nabla \mathrm{J}_{\mathrm{ad}}\right| \simeq \frac{J_{\mathrm{ad}}}{\lambda} \simeq \frac{\left(D / \lambda^{2}\right)\left(n-n_{\mathrm{e}}\right)}{a} .
$$

Thus, the diffusion dissipation of concentration is expressed by the equation $\dot{n} \simeq\left(D / \lambda^{2}\right)\left(n-n_{\mathrm{e}}\right) / a$.

On the other hand, the velocity of desorption of atoms $\int_{v} \dot{N} \mathrm{~d} v$ in volume $v$, based on the growth surface $s$, is as follows:

$$
\int_{v} \dot{N} \mathrm{~d} v=-\int_{V}(\nabla \mathbf{J}) \mathrm{d} v=-\int_{\bar{S}} \mathbf{J} \mathrm{d} \mathbf{s}
$$

where the first equation takes into account the continuity condition, while the second equation considers the Gauss theorem. As a result, the total change of concentration $n=n(t)$ near the growth surface is described by the equation

$$
\dot{n}=\frac{n_{\mathrm{e}}-n}{\tau_{n}}-J .
$$

At the same time, the characteristic relaxation time of the supersaturation is determined by the equalities

$$
\tau_{n} \equiv \frac{\lambda^{2}}{D}=\frac{\varepsilon}{4 \pi \sigma},
$$


the second equality being in agreement with the second relation (2.2).

Within the framework of the synergetic picture [8], the quasi-equilibrium condensation process is caused by the fact, that along with an increase of the supersaturation $n-n_{\mathrm{e}}$, the condensed atoms transfer the excess of their energy to the growth surface. As a result, its temperature $T$ (measured from the ambient temperature) increases as well. This enhances the evaporation of the deposited atoms due to an increase of the absolute value of the desorption flow $J<0$, which compensates the initial supersaturation.

Thus, an appropriate representation of the sequential picture of quasi-equilibrium condensation process requires a self-consistent description of the time dependence of the concentration $n(t)$ of adsorbed atoms, the growth surface temperature $T(t)$ and the desorption flow $J(t)$. According to [8], the evolution equations of these values contain dissipative components and the terms presenting positive and negative feedbacks, the balance of which provides a self-organization process. Thereby, in the equation (2.5), the first term on the right hand side represents the dissipation contribution, and the second term presents a linear relation between the rate of the concentration changes and the desorption flow.

The evolution equation for the temperature of the growth surface is presented in a similar way

$$
\tau_{\mathrm{T}} \dot{T}=-T-a_{\mathrm{T}} n J+\zeta(t),
$$

where $\tau_{\mathrm{T}}$ is a corresponding relaxation time, $a_{\mathrm{T}}>0$ is the coupling constant. In contrast to the equation (2.5), it is assumed that dissipation leads to the relaxation of the growth surface temperature to the value $T=\mathrm{d} 1$. The second term represents the nonlinear relationship of $\dot{T}$ with concentration and flow. Since the structure shown in figure 1 was obtained at an unstable temperature regime, the third term on the right hand side of (2.7) is a stochastic source of temperature changes representing the OrnsteinUhlenbeck process:

$$
\langle\zeta(t)\rangle=0, \quad\left\langle\zeta(t) \zeta\left(t^{\prime}\right)\right\rangle=\frac{I}{\tau_{\zeta}} \exp \left\{-\frac{\left|t-t^{\prime}\right|}{\tau_{\zeta}}\right\} .
$$

Here, $I$ is the intensity of temperature fluctuations, $\tau_{\zeta}$ is the time of their correlation.

To ensure self-organization, it is required to compensate the negative relationship in the expression (2.7) by a positive component in the evolution equation of the flow:

$$
\tau_{J} \dot{J}=-\left(J_{\mathrm{ac}}+J\right)+a_{J} n T
$$

where $\tau_{J}$ is a corresponding relaxation time, $J_{\mathrm{ac}}$ is the accumulation flow, $a_{J}>0$ is a constant of a positive feedback, allowing the growth of the $\dot{j}$ due to the mutual effect of the concentration of the adsorbed atoms and the growth surface temperature.

Thus, equations (2.5), (2.7), (2.9) present a synergetic system, where the supersaturation $n-n_{\mathrm{e}}$ is reduced to the order parameter, the temperature $T$ of the growth surface - to the conjugate field, and the desorption flow $J$ - to the control parameter [8]. As a result, the task is to investigate the possible stationary regimes in a stochastic plasma-condensate system, in particular, to consider the regime of the formation of porous structures.

The most simple investigation of the system [2.5), [2.7), [2.9] is possible within a dimensionless form using the characteristic scales for the time $t$, the concentration $n$, the temperature of the growth surface $T$, the flow $J$, and for the intensity of the temperature fluctuations $I$ :

$$
t_{\mathrm{s}} \equiv \tau_{n}, \quad n_{\mathrm{s}} \equiv a^{-2}, \quad T_{\mathrm{s}} \equiv \varepsilon, \quad J_{\mathrm{s}} \equiv \tau_{n}^{-1} a^{-2}, \quad I_{\mathrm{s}} \equiv \tau_{n}^{-1} a_{J}^{-2},
$$

where the above-mentioned length $a=\left(a_{\mathrm{T}} a_{J}\right)^{1 / 4}$ and energy $\varepsilon=\left(\tau_{n} a_{J}\right)^{-1}$ were used.

Thus, the dimensionless system of equations describing the fluctuational transition in a plasma-condensate system takes the form

$$
\begin{aligned}
\dot{n} & =-\left(n-n_{\mathrm{e}}\right)-J, \\
\epsilon \dot{T} & =-T-n J+\zeta(t), \\
\sigma \dot{J} & =-\left(J_{\mathrm{ac}}+J\right)+n T,
\end{aligned}
$$

\footnotetext{
${ }^{1}$ It should be noted that the condition $T=0$ does not correspond to the absolute zero since the temperature $T$ is measured from the ambient temperature.

${ }^{2}$ Investigation of the temperature fluctuation in the form of white noise was carried out in [2].
} 
where we introduced the relations for the relaxation times

$$
\epsilon=\frac{\tau_{\mathrm{T}}}{\tau_{n}}, \quad \sigma=\frac{\tau_{J}}{\tau_{n}} .
$$

\section{Statistical analysis}

While this system has no analytical solution, we will use the approximation $\tau_{n} \simeq \tau_{J} \gg \tau_{\mathrm{T}}$, which means that the temperature varies most rapidly. This situation is realized in the experiment very rarely, but the structure, presented in figure 1 is obtained exactly under unstable temperature regime (unstable cooling).

Then, on the left hand side of the equation [2.9], we can assume $\epsilon \dot{T} \simeq 0$, and the conjugate field is expressed by the equation $T=-n J+\zeta(t)$.

After some simple mathematical operations [8, 11, 12] the system (2.11) reduces to the evolution equation having a canonical form of the nonlinear stochastic Van der Pol oscillator [13]

$$
\sigma \ddot{n}+\gamma(n) \dot{n}=f(n)+g(n) \zeta(t) .
$$

Here, the friction coefficient $\gamma(n)$, the force $f(n)$ and the noise amplitude $g(n)$ are presented by the equations

$$
\begin{aligned}
& \gamma(n)=1+\sigma+n^{2}, \\
& f(n)=J_{\mathrm{ac}}-\left(n-n_{\mathrm{e}}\right)\left(1+n^{2}\right), \\
& g(n)=n .
\end{aligned}
$$

Then, the task is to find a distribution function of the system in the phase space formed by the concentration $n$ and the rate of its change $p=\sigma \dot{n}$ depending on time $t$.

To this end, the Euler equation (3.1) is conveniently represented by the Hamilton formalism

$$
\begin{aligned}
\dot{n} & =\sigma^{-1} p, \\
\dot{p} & =-\sigma^{-1} \gamma(n) p+f(n)+g(n) \zeta(t) .
\end{aligned}
$$

Thus, the above-mentioned probability density $P(n, p, t)$ is reduced to the distribution function $\rho(n, p, t)$ for the solutions of the system (3.3):

$$
P(n, p, t)=\langle\rho(n, p, t)\rangle_{\zeta}
$$

where $\langle\ldots\rangle_{\zeta}$ means the averaging over noise $\zeta$.

We will proceed from the continuity equation

$$
\frac{\partial}{\partial t} \rho(n, p, t)+\left\{\frac{\partial}{\partial n}[\dot{n} \rho(n, p, t)]+\frac{\partial}{\partial p}[\dot{p} \rho(n, p, t)]\right\}=0 .
$$

Further, the substitution of the equalities 3.3 leads to the Liouville equation

$$
\left[\frac{\partial}{\partial t}+\hat{\mathscr{L}}(n, p)\right] \rho(n, p, t)=-g(n) \zeta(t) \frac{\partial}{\partial p} \rho(n, p, t)
$$

where the operator

$$
\hat{\mathscr{L}}(n, p)=\frac{p}{\sigma} \frac{\partial}{\partial n}+\frac{\partial}{\partial p}\left[f(n)-\frac{\gamma(n)}{\sigma}\right] .
$$

Turning to the interaction representation [14]

$$
\varrho(n, p, t)=\mathrm{e}^{\hat{\mathscr{L}}(n, p) t} \rho(n, p, t),
$$

the equation (3.6) takes the form

$$
\frac{\partial \varrho(n, p, t)}{\partial t}=-\mathrm{e}^{\hat{\mathscr{L}}(n, p) t} g(n) \zeta(t) \frac{\partial}{\partial p} \mathrm{e}^{-\hat{\mathscr{L}}(n, p) t} \varrho(n, p, t) \equiv \varepsilon \mathscr{R}(n, p, t) \varrho(n, p, t),
$$


where $\varepsilon$ is a dimensionless small parameter [14]. Then, using the cumulant expansion method [15], one can obtain the kinetic equation 3

$$
\frac{\partial \varrho(n, p, t)}{\partial t}=\varepsilon^{2} \int_{0}^{t}\left\langle\mathscr{R}(n, p, t) \mathscr{R}\left(n, p, t^{\prime}\right)\right\rangle\left\langle\varrho\left(n, p, t^{\prime}\right)\right\rangle \mathrm{d} t^{\prime},
$$

neglecting the terms of $\varepsilon^{3}$ order [8].

Since a physical time $t$ is usually much longer than the noise correlation time $\tau_{\zeta}$, the upper limit of the integration can be set equal to infinity. Then, returning from the interaction presentation to the original presentation, for the distribution function (3.4) we obtain

$$
\left[\frac{\partial}{\partial t}+\hat{\mathscr{L}}(n, p)\right] P(n, p, t)=\varepsilon^{-2} \hat{\mathscr{N}} P(n, p, t)
$$

Here, $\hat{\mathscr{N}}$ is a scattering operator, which is given by the expression

$$
\hat{\mathscr{N}}=\left[M_{0}(t)-\gamma(n) M_{1}(t)\right] g^{2}(n) \frac{\partial^{2}}{\partial p^{2}}+\varepsilon M_{1}(t) g^{2}(n)\left[-\frac{1}{g(n)} \frac{\partial g(n)}{\partial n}\left(\frac{\partial}{\partial p}+p \frac{\partial^{2}}{\partial p^{2}}\right)+\frac{\partial^{2}}{\partial n \partial p}\right]+\mathscr{O}\left(\varepsilon^{2}\right),
$$

where $M_{0}(t)$ and $M_{1}(t)$ are the moments of the correlation function 2.8

$$
M_{i}(t)=\frac{1}{i !} \int_{0}^{\infty} t^{i}\langle\zeta(t) \zeta(0)\rangle \mathrm{d} t .
$$

From equation (3.13) one can obtain

$$
M_{0}(t)=I, \quad M_{1}(t)=I \tau_{\zeta} .
$$

Since, for this task, a complete distribution function $P(n, p, t)$ has a lower practical interest than its integral

$$
\mathscr{P}(n, t)=\int P(n, p, t) \mathrm{d} p,
$$

it makes sense to consider the moments of the initial distribution function

$$
\mathscr{P}_{i}(n, t)=\int p^{i} P(n, p, t) \mathrm{d} p .
$$

Then, the zero moment is reduced to the required integral (3.15).

Multiplying equation (3.11) by $p^{i}$ and integrating over all $p$, we arrive at the relation that can be written as a Fokker-Planck equation presented in the Kramers-Moyal form [16]

$$
\frac{\partial \mathscr{P}(n, t)}{\partial t}=-\frac{\partial}{\partial n}\left[D_{1}(n) \mathscr{P}(n, t)\right]+\frac{\partial^{2}}{\partial n^{2}}\left[D_{2}(n) \mathscr{P}(n, t)\right],
$$

where the drift coefficient

$$
D_{1}(n)=\frac{1}{\gamma(n)}\left[f(n)-M_{0}(t) \frac{g^{2}(n)}{\gamma^{2}(n)} \frac{\partial \gamma(n)}{\partial n}+M_{1}(t) g(n) \frac{\partial g(n)}{\partial n}\right]
$$

and the diffusion coefficient

$$
D_{2}(n)=M_{0}(t) \frac{g^{2}(n)}{\gamma^{2}(n)}
$$

are presented by the functions (3.2).

\footnotetext{
${ }^{3}$ It takes into account that the time derivatives in equations [3.6, 3.9] were treated according to the Stratonovich rule.
} 


\section{Stationary solution}

A stationary solution of the Fokker-Planck equation [16] yields a stationary distribution [8, 11]

$$
\mathscr{P}(n)=\frac{Z^{-1}}{D_{2}(n)} \exp \int_{0}^{n} \frac{D_{1}\left(n^{\prime}\right)}{D_{2}\left(n^{\prime}\right)} \mathrm{d} n^{\prime},
$$

where the partition function $Z$ is presented by the equation

$$
Z=\int_{0}^{\infty} \frac{\mathrm{d} n}{D_{2}(n)} \exp \int_{0}^{n} \frac{D_{1}\left(n^{\prime}\right)}{D_{2}\left(n^{\prime}\right)} \mathrm{d} n^{\prime} .
$$

The extremum condition for the distribution (4.1)

$$
D_{1}(n)-\frac{\partial}{\partial n} D_{2}(n)=0
$$

defines the stationary states of the plasma-condensate system.

Substituting expressions 3.18, 3.19, 3.2), and
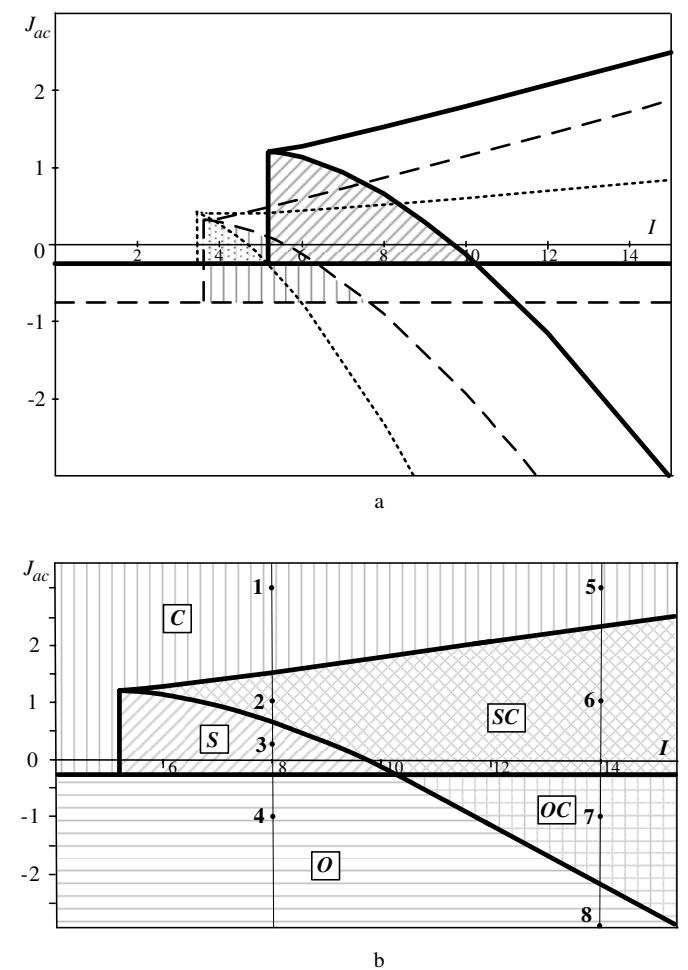

Figure 2. Phase diagram of the system. The solid line corresponds to $n_{\mathrm{e}}=0.25, \tau_{\zeta}=0.5$, the dashed line - to $n_{\mathrm{e}}=0.75, \tau_{\zeta}=0.5$, and the dotted line to $n_{\mathrm{e}}=0.25, \tau_{\zeta}=0.75$. The letters indicate the relevant domains of the phase diagram, and the dots (marked by numbers) correspond to the parameters at which the stationary concentration dependence (figure 3 ) is analyzed. the point $C^{\prime}$ characterized by a sufficient stationary concentration $n$. With a decrease of the accumulated flow (ray 2), there is observed a gradual disassembly of the previously formed condensate. This situation corresponds to the existence of two steady states with different concentrations (points $C$ and $S^{\prime}$ ). defining the stationary concentration dependence

$$
J_{\mathrm{ac}}=\frac{2 I(1+\sigma) n}{\left[(1+\sigma)+n^{2}\right]^{2}}+\left(n-n_{\mathrm{e}}\right)\left(1+n^{2}\right)-I \tau_{\zeta} n .
$$

Then, the condition that restricts the domain of the existence of the solution $n=0$ corresponding to the complete evaporation of the condensate from the growth surface, has the form

$$
J_{\mathrm{ac}}=-n_{\mathrm{e}} .
$$

The corresponding phase diagram of the system is shown in figure 2 ,

While figure 2(a) shows the effect of the system parameters (the equilibrium concentration $n_{\mathrm{e}}$ and the correlation time of fluctuations $\tau_{\zeta}$ ), figure 2 (b) considers in detail the domains of the phase diagram. In particular, the domain $C$ corresponds to the condensation process, the domain $S$ is characterized by the formation of porous structures, and a complete evaporation of the condensed matter takes place at the domain $O$. The domains, which are indicated by two letters, meet the coexistence of the above mentioned regimes.

It is more convenient to understand the processes occurring in each domain considering the example of the stationary concentration dependence presented in figure 3 .

Each point on the phase diagram [figure 2 (b)] corresponds to the ray in figure 3 (a), (b).

For example, for the ray 1 [figure 3(a)] only the condensation process is realized. It corresponds to (3.14) into the equation (4.3) we obtain the equation

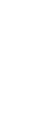



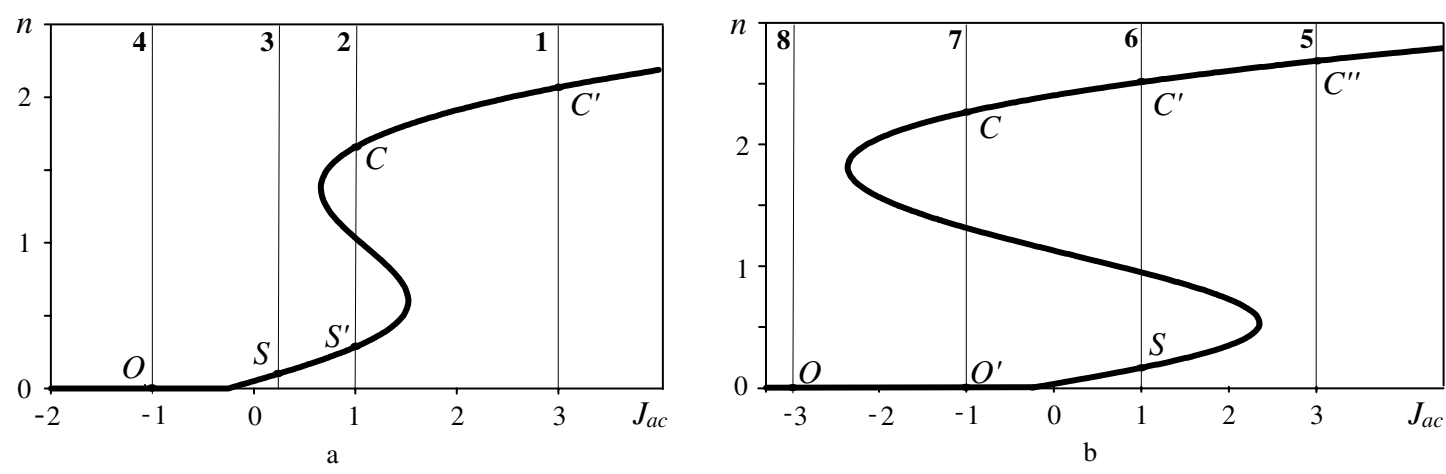

Figure 3. The dependence of the stationary concentration $n$ on the accumulated flow $J_{\mathrm{ac}}$ at $n_{\mathrm{e}}=0.25$, $\tau_{\zeta}=0.5$, (a) $I=8$, (b) $I=14$.

It should be noted that the additional intersection point of the main curve and the ray $\mathbf{2}$ (which is located between $C$ and $S^{\prime}$ ) applies to the non-physical plot and, therefore, it is not considered 4 . Turning to the state of the surface disassembly (point $S^{\prime}$ ), it is worth noting that in this case the usual evaporation of the upper layer of the condensate does not take place. First, the atoms which are less connected with the crystallization centers, are detached from the condensate surface. With a further decrease of the accumulated flow (ray 3 ), the only state of the surface disassembly remains (point $S$ ). This is the situation that characterizes the pattern shown in figure 1 and is of great interest to us. Going to the ray 4, the disassembly is replaced by the usual evaporation process (point $O$ ).

Analyzing the relationship (4.4) for higher intensity of fluctuations [figure 3 (b)], one can see that some changes occur. As previously, only condensation process (point $C^{\prime \prime}$ ) is realized for ray 5, while ray 6 is characterized by the coexistence of disassembly (point $S$ ) and condensation (point $C^{\prime}$ ) processes. The main difference is found for the ray 7, when together with the condensation process (point $C$ ), evaporation (point $O^{\prime}$ ) takes place. At the parameters specified for the ray 8, only evaporation occurs.

\section{Conclusion}

Based on the above analysis, we can conclude that processes occurring in the plasma-condensate system can be represented within the system (2.5), 2.7) and (2.9) describing the self-consistent behavior of concentration, temperature of the growth surface and desorption flow. Taking into account the fluctuations of the growth surface temperature with the correlation function (2.8) makes it possible to describe the most specific state (disassembly of the surface), when porous nanostructures may be formed. In addition, as shown in figure 2 (a), the system parameters have a significant effect on the domain of the formation of such structures. With an increase of the correlation time of fluctuations, this domain significantly decreases and shifts towards the lower values of the fluctuation intensity, while an increase in the equilibrium concentration results in a less significant decrease, as well as causes a shift along two axis (fluctuation intensity and accumulated flow). As a real experiment [1, 2], our theoretical approach has shown that the state of the surface disassembly is rarely realized. However, controlling the parameters of a system, we can reach the regime under which porous nanostructures are formed.

\section{Acknowledgements}

The authors are grateful to professor V.I. Perekrestov for placing the experimental material at their disposal.

\footnotetext{
${ }^{4}$ This also applies to the similar cases in figure $3(b)$.
} 


\title{
References
}

1. Perekrestov V.I., Olemskoi A.I., Kornyushchenko A.S., Kosminskaya Yu.A., Phys. Solid State, 2009, 51, No. 5, 1060; doi $10.1134 /$ S1063783409050266

2. Olemskoi A.I., Yushchenko O.V., Zhylenko T.I., Phys. Solid State, 2011, 53, No. 4, 845; doi $10.1134 /$ S1063783411040287

3. Olemskoi O.I., Yushchenko O.V., Zhylenko T.I., Prodanov M.V., Metallofizika i Noveishie Tekhnologii, 2010, 32, No. 11, 1555.

4. Olemskoi O.I., Yushchenko O.V., Zhylenko T.I., Ukr. J. Phys., 2011, 56, No. 5, 474.

5. Olemskoi A.I., Yushchenko O.V., Borisyuk V.N., Zhylenko T.I., Kosminska Yu.O., Perekrestov V.I., Physica A, 2012, 391, No. 11, 3277; doi 10.1016/j.physa.2011.10.027

6. Haken H., Synergetics, Springer, Berlin, 1978.

7. Olemskoi A.I., Theory of Structure Transformations in Non-Equilibrium Condensed Matter, NOVA Science, New York, 1999.

8. Olemskoi A.I., Synergetics of Complex Systems: Phenomenology and Statistical Theory, KRASAND, Moscow, 2009 (in Russian).

9. Pitaevskii L.P., Lifshitz E.M., Physical Kinetics, vol. 10, Pergamon Press, Oxford, 1981.

10. Landau L.D., Lifshitz E.M., Statistical Physics, part 1, vol. 5, Butterworth-Heinemann, Oxford, 1980.

11. Yushchenko O.V., Badalyan A.Yu., Phys. Rev. E, 2012, 85, No. 5, 051127; doi 10.1103/PhysRevE.85.051127

12. Khomenko A.V., Kharchenko D.O., Yushchenko O.V., Visnyk Lviv Univ. Ser. Phys., 2004, 37, 44.

13. Balanov A., Janson N., Postnov D., Sosnovtseva O., Synchronization. From Simple to Complex, Springer, Berlin, 2009.

14. Shapiro V., Phys. Rev. E, 1993, 48, 109; doi 10.1103/PhysRevE.48.109

15. Van Kampen N.G., Stochastic Processes in Physics and Chemistry, North Holland, Amsterdam, 2007.

16. Risken H., The Fokker-Planck equation, Springer, Berlin, 1987.

\section{Дослідження нанопористих матеріалів за умов квазирівноваги}

\author{
О.В. Ющенко, Т.І. Жиленко
}

Сумський державний університет, вул. Римського-Корсакова, 2, 40007 Суми, Україна

На основі трипараметричної системи Лоренца була розвинена модель, що дозволяє самоузгодженим чином описати поведінку системи плазма-конденсат поблизу фазової рівноваги. Враховуючи вплив флуктуацій температури ростової поверхні, були знайдені рівняння еволюції та відповідне рівняння ФоккераПланка. Побудована фазова діаграма, на основі якої визначені параметри системи, які відповідають режимові утворення пористих структур.

Ключові слова: самоорганізація, пересичення, фазова рівновага, конденсація, фазова діаграма 